Voix et Images

\title{
Les langues à Papineau : comment le texte national se fait littérature
}

\section{Alain Piette}

Volume 9, numéro 3, printemps 1984

Monique Bosco

URI : https://id.erudit.org/iderudit/200483ar

DOI : https://doi.org/10.7202/200483ar

Aller au sommaire du numéro

Éditeur(s)

Université du Québec à Montréal

ISSN

0318-9201 (imprimé)

1705-933X (numérique)

Découvrir la revue

Citer cet article

Piette, A. (1984). Les langues à Papineau : comment le texte national se fait littérature. Voix et Images, 9(3), 113-127. https://doi.org/10.7202/200483ar d'utilisation que vous pouvez consulter en ligne.

https://apropos.erudit.org/fr/usagers/politique-dutilisation/ 


\title{
Les langues à Papineau : comment le texte national se fait littérature
}

\author{
par Alain Piette, Université du Québec à Montréal
}

\begin{abstract}
En réalité nos idées viennent de France, mais nos mythes, nos fictions, nos cartes de crédit, notre confort, viennent des États-Unis. Au référendum c'est de cela qu'il s'agissait. Ceux qui ont voté avec leur tête ont choisi de dire oui. Ceux qui ont voté avec leur corps ont dit non. S'il est une leçon à tirer de tout cela, pour nous intellectuels, c'est qu'il faudra désormais proposer une nouvelle théorie et de nouveaux récits, dans lesquels désormais la tête et le corps seront réunisl.
\end{abstract}

Ce bilan postréférendaire de Jacques Godbout se présente de toute évidence, après la parution des Têtes à Papineau, comme un projet de fiction ou mieux comme un avant-propos au roman. Une fois de plus, le lecteur reconnaît la pertinence, pour la lecture d'un texte, de l'ouverture sur d'autres textes, contemporains ou antérieurs, qui recoupent celui-ci. Cette approche, particulièrement adaptée à un texte de Godbout, m'épargnera, je l'espère, la tentation de la lecture univoque, tentation d'autant plus grande ici que le commentaire de l'auteur semble baliser la lecture. Assurément, on voit mal comment on pourrait écarter de la discussion le rapport au référendum et au texte national. Mais à côté du discours sur le thème national, d'autres propos sont tenus : propos sur l'univers médical, sur le monde du spectacle, sur les média et enfin sur la littérature même. Il est possible d'entrevoir dès lors la complexité de ce roman : sous une armature narrative plutôt simple se joue un entrecroisement de discours dont j'essaierai de cerner les articulations diverses, en privilégiant l'étude des jeux multiples de l'énonciation et de leur impact sur les énoncés ${ }^{2}$. La première étape de ce parcours (les discours sur la médecine, le spectacle et les média) pourra paraître un peu longue au lecteur; sa fonction propédeutique se justifiera mieux par la suite : elle rend compte de la hiérarchie des propos du roman sans faire l'économie de la littéralité au profit de la seule lecture allégorique ou connotative. 


\section{De la tératologie...}

Sans être le plus important des discours qui tissent la trame du roman, le discours sur la médecine - discours souvent implicite - comporte assez d'autonomie pour être abordé ici. Il faudrait plutôt parler de critique de l'entreprise médicale puisque ce sont surtout les aspects négatifs de ce système qui sont exhibés ou dénoncés à travers les commentaires des narrateurs ou des personnages représentant le monde médical.

C'est sur l'univers médical même que s'ouvre le texte. La critique de la compétence médicale se laisse voir dans la caricature des limites intellectuelles du médecin. Les doutes jetés sur l'intelligence générale de Northridge finissent par ébranler la confiance en son intelligence proprement médicale. Des formules comme celles-ci suggèrent l'impuissance d'un diagnostic : «Nous sommes loin de même pouvoir nous poser la question» (p. 20), «Je ne comprends rien à tout cela» (p. 21), «Je ne saisis pas ce que vous voulez dire» (p. 23). Ces aveux d'impuissance (d'ignorance) sont corroborés par l'opinion des narrateurs : «Ả lire Northridge nous sentons pour la première fois peut-être que ces messieurs ne savent pas grand-chose encore sur le cerveau» (p. 133). La notion de prévisibilité scientifique apparaît comme le point névralgique de la compétence médicale : on ne peut en effet prédire de façon certaine ce qui résultera de l'opération. Aussi Northridge n'a-t-il plus qu'à constater en dernier lieu l'effet secondaire de l'opération, «a side effect of the brain operation» (p. 155). C'est ici la théorie qui est prise en défaut ainsi que "A.A.» le déplore dans son article de journal : "Au Centre de recherches en génétique l'on nous a répondu que l'enfant était impensable. Il faut croire que parfois la réalité fait rougir la théorie» (pp. 46-47). Dans cette perspective, il n'est pas dépourvu de sens que le docteur Pilotte soit un peu myope (p. 41) : pareil pilote ne peut mener qu'un bateau ivre à moins que ce ne soit un vaisseau-fantôme! Ce «brave garçon» (p. 41) est bien le collègue du docteur Bonvouloir au nom prédestiné. C'est dans la bouche de ce dernier que l'on trouvera la plus belle parodie du discours médical :

En réalité, si nous nous confinons à notre propre domaine, après des analyses approfondies de leurs types sanguins, après une cytologie complète, une étude des interactions biochimiques de leurs glandes endocrines et de leur organe de reproduction, nous devons admettre n'avoir rien trouvé d'atypique (p. 36).

Antithèse entre les prémisses et la conclusion : ce préambule hyperbolique semble une précaution oratoire apte à masquer l'insignifiance de la conclusion. Discours creux qui, à la limite, est presque une imposture.

Car c'est bien d'une imposture qu'il s'agit, celle de l'institution médicale tout entière : le discours des Têtes a Papineau se fait ici l'écho d'une critique tout à fait contemporaine (on reconnaîtra une fois de plus l'actualité du discours godboutien), critique dont le principal représentant est peut-être Ivan Illich avec sa Némésis médicale ${ }^{3}$. Dans cette imposante machine, le malade, il va sans dire, occupe bien peu de place : 
Elle (la garde) est persuadée de dominer la situation. Cela fait partie de son entraînement. C'est un avatar du métier. Tous les malades sont des facétieux. Il faut les mettre à leur place. Nous n'échappons pas à la règle (p. 121).

Au pire, le patient peut même être considéré comme un cobaye : «Nous sommes 'les Têtes à Pavlov!' se plaint François” (p. 118) ou comme un otage : «Northridge soutient que ce n'est pas de notre ressort, de toute manière (de décider de l'opération), rappelle François, nous appartenons à la science...» (p. 147). Tête à Pavlov ou prisonnier de la science, le patient est déshumanisé de toute façon. Il n'est donc pas étonnant que la technologie (l'ordinateur) s'immisce dans ses rapports avec le médecin. De ce point de vue, le roman ne dévalorise pas la technologie en soi, exaltée à travers le personnage de Marie Lalonde ${ }^{4}$ : il s'en prend plutôt à l'utilisation que l'institution médicale peut en faire. Le point culminant de l'ironie sur la technologie médicale est atteint dans le «texte opératoire» de François (pp. 143-145). Amalgame (!) de commentaire sportif, de science-fiction et de récit d'horreur (Allô police), ce texte surréaliste caricature le rôle de la technologie et la chosification du patient dans un système gravitant autour d'un ubricoleur sadique». Ce morceau choisi recoupe la description du docteur faite plus tôt par les narrateurs :

Il y a chez lui, quand il se déplie lentement pour se lever, un côté $\mathrm{Dr}$ Frankenstein dans le geste. Le front carré. L'oeil noirci. Il croit qu'à chaque problème correspond une solution. Il est persuadé que la technologie peut tout résoudre. Résoudre. François l'imagine dormant seul la nuit dans un laboratoire, bercé par le bouillonnement des cornues fumantes (p. 130).

Au «freak show»...

Faire voir le docteur Northridge en Frankenstein, c'est accéder au domaine de la représentation. Et c'est bien ainsi qu'on semble vouloir définir ici la pratique de la médecine, à savoir comme une mise en scène :

Nous allons tenter de prévoir les réactions de tout cet univers en équilibre. Et rédiger quelques scénarios. - J'ai toujours voulu être un acteur, lança François, mais j'aime moins l'idée d'un premier rôle dans un film d'horreur (p. 20)!

Cette scène d'horreur anticipée sera fictivement reconstruite (ainsi qu'on l'a déjà vu) par François lui-même à la fin du roman : on y trouve projecteurs et masques comme au théâtre (p. 143). Spectacle aussi que la naissance des deux frères à la page 41 :

Ils (les infirmières et le médecin) nous offraient les masques de la peur et du rire. Les accouchements sont des sacrés coups de théâtre! L'enfant déchire le rideau de chair et c'est la première! Applaudissons.

Peut-on d'ailleurs concevoir la pratique médicale d'une autre façon lorsque le patient est un monstre, que son être physique est en soi un spectacle et que jouer un rôle lui est en quelque sorte congénital? C'est alors que le monde réel même devient représentation. 
Cette problématique explique l'importance accordée à tous les arts visuels dans les Têtes à Papineau. Représentations de tous genres des "Têtes» : statuettes (pp. 17 et 21), courtepointes et meubles (p. 31), et surtout photographies (pp. 31, 97, 107, 112, 124). Plus encore que l'image fixe, c'est le spectacle qui sera évoqué dans l'univers du roman. Allusions au monde du spectacle dans ses manifestations les plus diverses (théâtre, cinéma, télévision, show-business, etc.), allusions trop nombreuses pour être toutes citées mais dont on peut fournir quelques échantillons : Marie Lalonde jouant Phèdre, Andromaque et Aurore (p. 43), la «Visite des Acteurs» (p. 52), la soirée des Oscars à la télévision (p. 66), «A.A.» en costume de Gene Autry (p. 97), les débuts prometteurs de la chanteuse Marie Lalonde (p. 102), etc. C'est parfois toute une séquence qui impliquera l'univers du spectacle comme c'est le cas avec Irma Sweet et Dippydou.

Mais dans ce monde de fantaisie qui contraste singulièrement avec la régularité scientifique et technologique, le roman s'attarde de façon particulière - et pour cause - au cirque et au freak show, c'est-à-dire là où le masque est un don de la nature. Cela commence avec la visite des curieux à l'hôpital (p. 45). Mais c'est surtout au chapitre "cinquièmement" que ce motif est développé et valorisé : "Nous ne nous sommes sentis «chez nous», pendant longtemps, que dans la grande maison de la famille Fontaine» (p. 77). Jamais les narrateurs ne porteront un jugement négatif sur ce monde, le monde du Racine Greater Show, celui des monstres. Le chapitre se terminera sur la grand-mère, véritable casse-tête vivant, mais combien sympathique! Les performances des «Têtes» se multiplient à l'université («Freak show. Fric chaud., p. 106) jusqu'à l'apothéose de la remise des diplômes, spectacle comparable à celui de la naissance (p. 111), et l'exhibitionnisme se poursuit avec le talk show (p. 112). Même l'opération finale est une reconstitution de la Passion (p. 146). "The show must go on».

\section{En passant par les médias...}

Des «Têtes» à Papineau comme spectacle à la recherche du spectaculaire par les médias il n'y a qu'un pas, vite franchi par le «romanjournal». On fera allusion à des magazines friands de sensationnalisme :

Vous connaissez certainement cette photographie célèbre (le directeur du musée est à quatre pattes et nous fait risette) publiée dans le magazine Life (p. 31).

Prenant charge des entrevues, "A.A.» nous recommanda d'éviter les journaux à sensations pour n'accepter de sollicitations que des revues assises (...) Il y eut une seule exception, pour Paris-Match (p. 107). 
Le texte n'entretient pas ici d'équivoque sur l'orientation de ce célèbre magazine, orientation si bien caricaturée jadis par le Paris-Flash des Bijoux de la Castafiore. Ailleurs, la perspective s'élargira jusqu'à viser l'ensemble des journalistes :

Quand ceux-ci avaient découvert que Marie Lalonde était à nouveau enceinte, ils en avaient profité pour lancer le Grand Concours du Bicéphale. Ils avaient annoncé un prix grandiose, à l'occasion du congrès mondial des jumeaux d'origine, à celle ou celui qui prédirait le sexe du prochain monstre (p. 54).

La satire atteint ici un sommet. Non seulement la presse crée l'aspect sensationnel de l'événement par anticipation, mais encore le congrès luimême et la récompense s'avèrent une fumisterie totale : existe-t-il d'autres catégories de jumeaux que celle des jumeaux d'origine? Et ce prix «à celle ou celui qui prédirait le sexe du prochain monstre» se doit d'être "grandiose» puisque cette prédiction implique le choix entre deux seuls éléments et qu'il y aura donc nécessairement des milliers de gagnants! L'imposture des journalistes organisateurs du «Concours» paraît à son comble lorsque nait l'enfant : il n'y a même pas un seul gagnant puisqu'il n'y a pas de monstre. Il ne s'agit donc pas de ce qui peut «titiller la presse» (p. 123), mais bien de ce que la presse peut faire pour titiller le public et, ce faisant, pour le tromper.

La critique s'étendra enfin à tout le domaine de l'information :

Les travailleurs de l'information nous avaient redécouverts. Non seulement nous étions bicéphales mais aussi étions-nous intelligents. "Un jeune monstre sur la montagne», titrait un quotidien du soir parlant de notre arrivée à l'Université Montis Regii. Enfin. La nouvelle fut reprise de Singapour à Yaoundé (p. 107).

Cette rapacité des média et particulièrement des journalistes en face de la nouvelle prête à une ironie subtile lorsque Charles lance au beau milieu d'un groupe de journalistes à la maison paternelle : «Hahem. Nous avons une grande nouvelle à vous annoncer ...» (p. 67). Si le texte souligne nettement cette tendance du journalisme à rechercher l'effet de la nouvelle (du moins le journalisme de la catastrophe, dirait Godbout), s'étonnera-t-on alors de la métamorphose qui surviendra dans la vie professionnelle du père des Papineau :

Freak show. Fric chaud. François décréta que nos têtes étaient notre talent et que cela devait se monnayer. "A.A.», puisqu'il était aussi journaliste, ferait office d'impresario (p. 106).

C'est ainsi qu'on passe du monde de l'information à celui du spectacle non sans avoir tenu auparavant la chronique artistique (p. 59).

Le lien constant qui s'établit entre l'information et le spectacle à partir du sensationnel fonde le caractère superficiel du journaliste; littéralement happé par les événements-spectacles, il n'a pas beaucoup le temps de faire le point : 
"A.A.» est la preuve vivante de la théorie des psychologues du bonheur : il ne s'exprime pas pour dire quelque chose, il décèle ce qu'il avait à dire en le proférant. Il ajoute qu'être journaliste dix heures par jour lui suffit. Dans son métier il ne peut se permettre de penser. Il cueille, il formule, il retransmet. Il aurait tout aussi bien pu être plombier (p. 65).

Les fils Papineau prendront la relève de leur père ${ }^{5}$ :

Entre nos cours à l'Université, auxquels il fallait tout de même faire acte de présence, nos conférences et nos colloques, nos apparitions à la télévision, et notre vie sociale, il nous restait peu de temps pour réfléchir. C'est peut-être ce qui nous sauva. Nous savions planer à la surface des idées, des gens et des choses. Surfistes des vernissages, piliers des cocktails de lancement, notre abattage ne souffrait aucune comparaison. (...) Spirituels et superficiels (pp. 110-111).

Cet aveu des narrateurs évoque un air connu :

En ce sens, plus radical, l'œeuvre de Godbout est superficielle : elle ne veut avoir affaire qu'aux surfaces, aux lieux, à ce qui les enclôt (les murs) et aux routes qui permettent d'y arriver ou d'en sortir, à ce qui se touche - «Godbout touche à tous» - , se détériore et se remplace ${ }^{6}$.

Etrange chaîne intertextuelle où les narrateurs semblent avoir pris connaissance de la critique de Marcotte sur l'auteur Godbout. Mais alors cette reprise ne peut être qu'ironique lorsqu'on sait (par le contexte d'énonciation) que Godbout ouvre lui-même dans le monde des communications et que Marcotte fut critique journalistique. Serait-ce à dire qu'il faudrait revoir le portrait caricatural du journaliste dans les Têtes à Papineau et se demander s'il ne s'agit pas d'un discours ironique sur un stéréotype, celui du journaliste superficiel :

Papa a souvent aimé inventer des histoires et maman ne l'a jamais contredit. Il possède ce que François appelle un «tempérament de reporter». Il entend par là une propension non pas à maquiller la vérité ou à mentir bassement, mais à produire de nouvelles réalités (p. 32).

Cette conception du journalisme (du reportage) est partagée - et sans ironie cette fois - par l'essayiste Godbout :

Donc les praticiens de l'information s'imaginent qu'ils peuvent être les honnêtes courtiers de la réalité. Or il est bien évident qu'une information n'est pas un reflet de la réalité, mais la production d'une nouvelle réalité. La preuve en est qu'il n'y a nouvelle que s'il y a un journaliste. C'est le journaliste qui produit la nouvelle, comme le romancier produit son roman?

Ce qui m'intéresse plus encore, c'est que l'information est une forme de transformation de la réalité, et que je retrouve dans l'information des rapports que j'établis dans le roman ${ }^{8}$. 
Godbout s'en prend ici à un autre stéréotype, celui de l'objectivité du journalisme. Ironiquement, il démontre dans son dernier roman que le roman peut être un journal (avec le jeu de mots obligé) sans devenir pour autant superficiel (il s'agit d'un journal intime). Mais par cette réflexion sur la littérature, j'anticipe sur un développement ultérieur. Il faut au préalable discuter cette épineuse question du rapport que le texte établit avec la réalité, question soulevée par Godbout dans les textes cités plus haut.

\section{Vers un nouveau texte national $9 . .$.}

Le lecteur atteint ici une strate supérieure du texte. Sans doute, l'autonomie relative des trois discours traités précédemment a été établie. Mais ces trois matériaux habilement «amalgamés» ne constituent que l'infrastructure du texte : les propos les plus essentiels ne sont pas là. Le texte est constamment sollicité par un hors-texte; on y parle des réalités politique, culturelle, linguistique du Québec de façon explicite et implicite, au niveau du dénoté et du connoté, on y parle de la nation québécoise :

Un sujet aussi inévitable que la problématique de la langue ou le pénible portrait de l'homme colonisé canadien-français (p. 27).

D'une cour à l'autre, d'un juge à l'autre, personne n'osait statuer sur notre appartenance (p. 53).

Les Québécois, depuis la bataille des Plaines d'Abraham, veulent gagner partout à la fois. Ils achètent des billets de toutes les loteries. Ils auraient élu une tête à Québec, et l'autre à Ottawa! L'idéal. Puisque Charles parle anglais «sans accent» ne sommes-nous pas un parfait bicéphale bilingue (p. 96)?

Le sujet préféré des organisateurs de colloque? L'autonomie (p. 110).

Ce discours sur le thème national est un discours nationaliste qui s'inscrit dans la tradition à maints égards. Il reprend tout d'abord la vieille opposition entre Anglais et Français. Cette dichotomie se retrouve chez les frères Papineau en premier lieu. Le pôle anglophone s'élargit pour les besoins de la cause jusqu'à inclure les Américains :

Charles trouve ces jeux de mots particulièrement idiots. Le côté gaulois de François l'horripile. Il soutient que si les Français aiment tant les jeux de mots c'est que leur langue est constamment surveillée. Il préfère l'approche américaine. Les jeux de guerre et de hasard. Il dit que les Anglais ont de l'esprit. "Le calembour n'est pas un trait d'esprit. Ả preuve», dit souvent Charles en se renfrognant, «ça ne fait pas rire les enfants» (p. 17).

Intéressant discours sur un stéréotype (encore une fois chez Godbout!), soit l'opposition séculaire entre l'esprit français et l'esprit anglais, mais aux dépens de celui-ci comme en témoigne l'argumentation dérisoire de' Charles. Un détail attire l'attention du lecteur, détail qui révèle un trait récurrent dans le personnage de Charles : «en se renfrognant». Cela inaugure une longue liste de qualifications mettant en évidence son caractère insuppor- 
table : «renfrogné» (p. 62), «buté» (p. 63), "Charles bougonnait» (p. 65), «cela horripile Charles» (p. 119), «J'ai de plus en plus la tribu en horreur, répondit Charles. Puis, il cracha au loin de dépit (...)» (p. 130), «avec une moue de mépris» (p. 145). On peut ajouter à cette série le passage où Charles fait de l'obstruction dans le travail de rédaction commune (p. 64), l'allusion de François à l'anthropophagie de son frère (p. 129), le rôle de Judas tenu par Charles dans la reconstitution de la Dernière Cène (p. 146), etc. Le bilan offre une image négative de l'anglophone à travers Charles, non sans un certain paradoxe au niveau de l'énonciation puisque Charles est un des deux narrateurs. Ce parti pris évident des narrateurs qui rend Charles antipathique aux yeux du lecteur débouche logiquement sur la lettre finale en anglais ${ }^{10}$, après la maxime annonciatrice du chapitre précédent : "L'évolution, c'est la raison du plus fort» (p. 150). Ainsi se réalise la double conjonction «dominé-sympathique» et «dominant-antipathique».

Mais l'opposition traditionnelle se présente aussi à un autre niveau où Charles-François Papineau symbolise le Québec : la marginalité biologique est métaphore de la marginalité culturelle, le bicéphale s'oppose à l'unicéphale. Dans cette opposition, l'Anglais n'est pas toujours valorisé, bien au contraire : «Des milliers de morons unicéphales» (p. 45). Bien que le sens littéral ne désigne pas ici l'anglophone, tous les autres passages où le terme «unicéphale» est employé contraignent à y lire aussi cet autre sens.

L'irréductible antinomie se révélera enfin à travers l'image de la grenouille :

C'est notre image publique la plus répandue (p. 17).

Dans l'océan anglophone tout ce qui ne saxonne est un batracien (p. 123).

Comment une grenouille pourrait-elle nager dans une mer d'unicéphales (pp. 150-151)?

Cette astucieuse représentation romanesque d'un symbole de mépris bien connu s'offre comme relais littéraire aux connotations péjoratives du terme «frog». On notera cependant que la charge négative de l'image est beaucoup moindre ici que dans la réalité québécoise. Cela tient en bonne partie à la présentation que les narrateurs-monstres font d' eux-mêmes : ils rendent sympathique ce "monstre sorti de l'océan» (p. 30), cet "animal préhistorique», ce «serpent de mer» (p. 124)" ${ }^{11}$. Cette bête rappelle singulièrement celle de l'Isle au dragon. Mais il s'est produit une transformation partielle des rôles : alors que là le dragon représentait aussi l'ennemi à abattre (du moins dans une partie du roman), l'anglophone ou mieux l'Américain, ici le mal ou le danger est davantage intériorisé, ce qui donne le beau monstre, la «Belle Bête» (pour jouer de l'intertexte). Le référendum de 1980, survenu entre les deux romans de Godbout, justifie assez bien 
cette transformation. Plus que jamais, le Québécois apparaît ambivalent, d'où l'ambivalence du monstre: «Notre monstruosité les fascinait» (p. 130). Cette vision autocritique du Québécois n'est-elle pas une révision du discours nationaliste dont il a été question plus haut? Encore plus que l'Isle au dragon, ce roman voudrait donc proposer, à côté des thèmes connus, de nouveaux horizons.

Si l'aspect négatif de la figure de Charles a déjà été souligné, on n'a pas encore parlé des traits négatifs de François, le "gaulois». Le texte tient à présenter cette contrepartie, même si François est mieux vu que Charles des narrateurs (toujours selon le même paradoxe). Il est tout d'abord décrit comme un être superficiel qui agit avant de penser : "Il préfère l'action immédiate, quitte à se brûler les pattes (...) François, s'il n'est pas stimulé de l'extérieur, ne pense à rien» (p. 15). Au niveau de la parole, cette tendance fait de François un verbo-moteur : «quand François phrase il s'écoute, il frappe les mots comme des cymbales» (p. 21). Enfin, le mélo s'accorde bien à ce caractère superficiel (p. 64), alors qu'on imagine plutôt le vrai lyrisme du côté de Charles.

La répartition des paradigmes Anglais/Français ne correspond pas toujours aux stéréotypes culturels et aux canons du discours nationaliste traditionnel. Comment, sous ce rapport, François peut-il fréquenter les H.E.C. (associées au monde des affaires) et Charles, la Faculté des Lettres? Plutôt que de souligner un état de fait dans la société québécoise réelle, cela ne veut-il pas suggérer de nouvelles voies, comme si le salut national se situait dans une répartition différente des fonctions sociales? Cette répartition devient plus évidente encore lors du référendum où la mère, qui travaille dans l'informatique, est associée à François (jusqu'à la page 151) et le père, journaliste, à Charles ${ }^{12}$. Mais peut-être faudrait-il scruter de plus près les traces, dans le roman, d'un événement qui paraît être au centre du contexte d'énonciation de ce texte.

La première constatation d'importance vise le résultat même du référendum : c'est le "roui» qui l'emporte dans le roman, c'est l'amalgame, c'est le changement, contrairement au référendum historique de 1980. D'autre part, les appuis au «oui» viennent des éléments identifiés comme anglophones avec une exception notable, François. Cette défection de dernière minute (p. 151) évoque singulièrement les événements de 1980 , alors que des indécis ont vraisemblablement eu peur d'appuyer le «oui» à la dernière minute! Autre brouillage intéressant, la position des deux têtes (p. 23) : la tête de Charles est à droite même s'il prône le changement. Ne voudrait-on pas ébranler ici le caractère rigide et artificiel des stéréotypes où nous (Québécois) nous enfermons, soit la série «Non - pro-anglophone droite - statu quo (fédéralisme renouvelé)» et la série opposée "Oui francophone - gauche - changement (souveraineté-association)»? C'est bien une révision du discours nationaliste des années 70 qui est proposée à travers l'allégorie romanesque du référendum. 
Le texte ne se limite pas cependant à offrir de nouvelles avenues : il tient aussi à marquer son refus de certains thèmes de la vieille idéologie nationaliste. Cela est patent dans le chapitre «cinquièmement» : on y trouve un véritable "foyer idéologique», selon l'heureuse expression de Philippe Hamon ${ }^{13}$. Ce chapitre s'ouvre sur un premier signal: "chez nous», expression qui connote l'idéologie nationaliste, citation abrégée du célèbre "Maîtres chez nous» (on notera d'ailleurs les guillemets du texte-tuteur). Le signal est réitéré deux pages plus loin (p. 79) sous une forme équivalente : «les nôtres» (on notera ici encore les guillemets).

Puis, nouveau signal, une citation du docteur Bonvouloir à propos des difficultés d'adaptation des monstres se termine par la phrase : «Ils savent qu'ils ne sont pas de ce monde" (p. 80). Cette paraphrase de l'Évangile selon saint Jean évoque le thème du messianisme de notre vieux texte national. Cette évocation appartient au registre de la parodie comme le confirme la suite du texte :

Et puis tout ça était, pour ainsi dire, inévitable! Quelques centaines de familles françaises à l'origine, on couche ensemble cousins cousines pendant les longs hivers québécois et voilà six millions de descendants quelques siècles plus tard. Descendants.

«C'est qu'on s'aimait par chez nous. On se lâchait pas. On se tétait les oreilles en famille», dit Charles que toute évocation ancestrale fait frémir.

«C'est tout de même ainsi», répondit François, «que nous avons conservé nos traditions, notre langue, notre foi, nos chansons et nos chromosomes. Chrysostome!»

C'est ainsi que le Dr Bonvouloir a dénombré dans nos familles plus de deux cents maladies de dégénérescence. (...) C'est ce que M. Fontaine appelait «les richesses naturelles infinies de la province québécoise». (...) Nous sommes assez riches pour occuper des milliers de congrès médicaux. (...) Nous sommes riches (pp. 80-81)!

Phénomène unique dans ce roman : le «nous» ne désigne pas que les personnages-narrateurs, mais inclut ici toute la collectivité québécoise au sens littéral (sans métaphore ou allégorie). Il alterne d'ailleurs avec le «on», outil usuel de la langue parlée, comme pour souligner encore davantage l'extension référentielle du "nous». De plus, le «on» se trouve dans le discours de Charles où la vie de famille est tournée en dérision. À l'opposé, la réponse de François associera le «nous» du français standard à la conservation des valeurs nationales; mais ce discours sérieux se convertira aussi en parodie par l'ajout des «chromosomes» à la série culturelle et par la rime bouffonne du juron québécois "Chrysostome». Il faut relever l'exception constituée par cette rime, ce qui la met en évidence : en effet, le 
procédé mis en œuvre dans les Têtes à Papineau est la reprise du dernier mot de la phrase («les choses répétées plaisent», p. 109), mais dans le cas de «chromosomes» il n'y a qu'une reprise phonique. Quelques lignes plus haut, il y avait la reprise ironique de "descendants", dont le jeu de mots cligne de l'oeil à "dégénérescence». Ainsi, dans ce "carrefour normatif» il s'agirait, entre autres, du procès ironique du vieux thème de la survivance : «Elle (maman) nous a tout appris de la lutte pour la survie» (p. 82).

Ce discours critique s'appuie - on reconnaît une fois de plus l'actualité du discours de Godbout - sur des observations faites par nombre de médecins-chercheurs ${ }^{14}$. Cette base scientifique permet une révision de textes classiques comme celui-ci :

(...) ici toutes les choses que nous avons apportées avec nous, notre culte, notre langue, nos vertus et jusqu'à nos faiblesses deviennent des choses sacrées, intangibles et qui devront demeurer jusqu'à la fin ${ }^{15}$.

Cette révision du passé est un nouvel aide-mémoire («Je me souviens») :

Quand nous sommes retournés sur les lieux de notre enfance le long des rives du fleuve, tout nous est apparu plus triste, plus délabré, plus misérable que le souvenir que nous en avions. Même les nains peuvent rapetisser (p. 90).

La critique vise même le nationalisme des années 70 (il en a déjà été question) tel que véhiculé par le Parti Québécois et son chef, René Lévesque. On pense nécessairement à ce dernier avec le thème du "chez nous» joint à celui des «richesses naturelles» : on peut y voir comme un appel à plus d'autocritique et de discernement.

Enfin, la problématique nationaliste n'était-elle pas déjà inscrite dans le titre même de l'ouvrage? Quel programme s'y trouvait-il donc? Pour répondre adéquatement à cette question, il faut partir du matériau brut qu'y travaille Godbout, soit l'expression figée concernant Papineau. Le cliché bien connu ${ }^{16}$ se présente sous la forme suivante : "C'est pas la tête à Papineau» ou sa variante : «Faut pas se prendre pour la tête d̀ Papineau». Dans les deux cas, la forme est négative, la première étant carrément une litote pour désigner une intelligence limitée, la seconde, une sentence évoquant la grenouille (comme par hasard!) de la fable. De toute façon, il s'agit d'une mythification du passé cristallisée dans le personnage de Papineau qui semble être le seul à pouvoir occuper le pôle positif des valeurs intellectuelles. Le titre du roman de Godbout subvertit totalement ce donné initial. Tout d'abord, le pluriel ${ }^{17}$ centre l'attention du lecteur sur la collectivité plutôt que sur l'individu Papineau. Ensuite, la négation a disparu : il ne subsiste qu'un groupe nominal ouvert à toutes les possibilités au niveau des qualifications ou des actions. 


\section{Ou sa version littéraire}

C'est donc en polarisant l'attention du lecteur sur le matériau de la langue qu'on veut ici lui parler du texte national. Cette focalisation sur le signifiant révèle que la problématique de l'écriture et de la littérature sera au cour du texte. Il ne manque pas en effet d'allusions aux textes littéraires dans les Têtes à Papineau. On y mentionne Kafka (p. 27), Rimbaud (p. 66), Cendrars, Eluard, Prévert (p. 109). On y fait allusion à Voltaire : «cultiver leurs arpents enneigés» (p. 88) et à la Bible : «le lait et le miel» (p. 128), connotant par là les contradictions de la société de référence. Mais surtout, on y parle de littérature populaire, de littérature pour enfants et adolescents, de contes et de légendes : «Perrault, Dickens, Melville, Anderson, la Bibliothèque rose et la bleue» (p. 59); «l'Almanach» (p. 97); "D'Artagnan et Aramis» (p. 136). On y évoque l'île de Robinson (pp. 61-62) ou celle de Calypso (p. 135). Cette insistance sur la littérature populaire relaie à sa façon la conception que se fait Mireille de la littérature dans D'amour, P.Q.

Mais plus qu'un simple vade-mecum de l'histoire littéraire, les Têtes $\grave{a}$ Papineau représentent l'acte même d'écrire. L'instance narrative est assumée par les deux têtes (p. 28) :

L'essentiel, crois-moi, c'est d'être confrontés dans la même phrase jusqu'à la phase finale (p. 64).

Chacun des mots que nous enregistrons doit être approuvé par les deux têtes qui gouvernent (p. 119).

L'utilisation du pronom «nous» pour désigner un double narrateur en acte d'écriture me paraît un phénomène unique dans la littérature québécoise. Le roman qui s'en approche le plus est l'Hiver de force de Réjean Ducharme. Mais à y regarder de plus près, André en est le seul narrateur puisqu'il parle de Nicole sur le mode du «elle». Le «nous» de ce roman ne désigne donc que le couple de l'histoire. Dans les Têtes à Papineau, le «nous» renvoie aussi à l'instance narrative et chacune des têtes est désignée (comme l'était Nicole) par la troisième personne.

Cette coordination difficile dans la production du discours en évoque une autre : «En réalité», comme l'a expliqué François à Northridge, «organisés comme nous le sommes, une simple masturbation demande une rencontre au sommet des volontés» (p. 73). Ainsi, sexe et texte vont de pair dans les Têtes à Papineau. Cette homologie semble même jouer au niveau de la technique. "C'est un récit bi-graphique» (p. 28), disent les narrateurs. L'affirmation est on ne peut plus claire, malgré le jeu de mots sur «biographique». Quant à cette autre affirmation : "Quand Charles écrit, François parle» (p. 96), sa portée n'est que contextuelle. On mentionne à deux reprises le fait qu'ils écrivent les bras croisés (p. 95, p. 121). Le texte, 
sans être explicite toutefois, veut donc suggérer qu'il y a deux rédactions concomitantes préliminaires au texte final. Il n'en va pas autrement de leurs prouesses amoureuses : la manipulation et la succion des seins d'Irma Sweet (p. 128) ne sont pas pensables sans que les bras se croisent. C'est ainsi qu'«au pays de Québec» on fait l'amour, comme on écrit, les bras croisés!

Il n'est pas étonnant alors que «les discours se croisent, se bousculent, s'entrechoquent" (p. 119). La difficulté, pour les deux têtes, d'écrire ce texte collectif rappelle grandement la difficulté d'écrire le texte national, comme en témoigne le passage suivant :

Nous sommes, pour' ainsi dire, idéologiquement séparés. C'est pourquoi ce livre ne peut être un effort de raccordement. Une médiation. Au contraire nous le voyons comme l'exercice lucide, utile et difficile de nos libertés. Il n'est plus question de tenir des discours chacun de notre côté. Ce n'est plus le temps des élections (p. 29).

C'est donc la difficulté d'être ensemble, au niveau de l'histoire racontée, qui se réflète sur la narration commune. L'issue de cette impasse est la lettre finale postopératoire, sur laquelle il convient maintenant de s'arrêter.

La lettre est adressée aux Éditions du Scorpion et annonce la rupture du contrat initial avec l'éditeur. Ce fait souligne les implications pratiques de la littérature avec l'institution littéraire. Une critique s'était déjà manifestée à l'endroit de l'éditeur (p. 27): les narrateurs y dénonçaient sa tendance au sensationnalisme (dénominateur commun avec les média!). Mais le résultat sensationnel de l'opération ne pourra être raconté. C'est bien l'impossibilité pour la littérature de se réaliser qui est signifiée ici, plus précisément pour une littérature d'expression française ${ }^{18}$. Le motif allégué est donc d'ordre linguistique, ce qui affirme la dépendance de la littérature vis-à-vis de son matériau. Élément significatif, le long discours français des narrateurs ne compte aucun verbe performatif, alors que la petite lettre en anglais de Charles-F. Papineau en compte un : «I promise you» (p. 156), ce qui reconnaît à la langue anglaise un statut spécial au niveau du faire ${ }^{19}$.

Mais il faut pousser la réflexion plus avant et considérer la forme même de ce court texte anglais. 11 s'agit d'une lettre d'affaires (genre protocolaire)! Cela l'oppose fortement au reste du texte qui est défini comme un journal intime (pp. 28 et 117). Ainsi, ironiquement, la seule production de l'ex-étudiant en Lettres est une lettre! Par ailleurs, il subsiste un paradoxe non résolu: le journal intime est rédigé à la première personne du pluriel, alors que la lèttre officielle s'exprime à la première personne du singulier. C'est la contradiction même que la collusion des genres opposés 20 veut signifier et ce nouveau signifié englobe le précédent (sur l'impossibilité de la littérature) : la littérature existe tout de même (le roman de Godbout est là devant moi), mais elle n'existe pas sans contradiction et sans paradoxe, tout comme la société québécoise (l'on revient au point de départ, le référendum). 
Il serait illogique de conclure sur ce roman du «nous» en se limitant à l'aspect sémantique: l'aspect pragmatique se doit d'être aussi envisagé. «Je ne crois pas aux traces, dit Charles, nous avons payé assez cher, il me semble, notre filiation aux Papineau» (p. 22). Je ne peux lire pareille phrase sans y voir l'allusion au passé des Québécois, mais je donne alors au «nous» une extension que n'a pas le sens littéral: je comprends «nous, Québécois». Ainsi, c'est le contexte d'énonciation seul qui détermine l'implication particulière des lecteurs vis-à-vis de ce texte (des lecteurs étrangers ne pourraient s'incorporer au «nous»). L'effet de lecture est donc d'associer le lecteur québécois francophone au journal intime et de le dissocier de la lettre finale (l'anglais servant de repoussoir). Par le fait même, c'est une prise de position ferme au plan linguistique que le texte suggère et cela le situe au coeur du débat référendaire, mais en radicalisant et en spécifiant les enjeux. Ainsi, le couteau est toujours sur la table, mais les points de suspension n'y sont plus. "Les Canadiens-français étaient doux» (p. 85): laisse-t-on entendre par l'emploi de l'imparfait que l'ère de la tolérance est révolue pour les Québécois? Ou peut-être est-ce une stratégie ultime du «romancier du discours social» avant que nous, Québécois, ne soyons amalgamés pour de bon:

C'est le sort qui attend les francophones de l'Amérique du Nord, dans le contexte actuel. Je pense que nous allons basculer du côté américain avec une vitesse "grand $V$ ». Malgré toutes les lois, malgré tous les efforts $^{21}$.

1. Jacques Godbout, «Les bons sauvages», Liberté, $\mathrm{n}^{\circ}$ 131, septembre-octobre 1980 , p. 11.

2. Je renvoie le lecteur à l'ouvrage de Dominique Maingueneau, Initiation aux méthodes de l'analyse du discours, problèmes et perspectives, Paris, Hachette, «Langue. Linguistique. Communication», 1976. Le long chapitre sur l'énonciation en particulier $m$ 'a été fort utile, ainsi que les textes de Benveniste sur cette question: Problèmes de linguistique générale, Paris, Gallimard, 1966, partie V, et «L'appareil formel de l'énonciation», Langages, 17, mars 1970, pp. 12-18.

3. Paris, Éditions du Seuil, «Points», 1981.

4. Contrairement au père qui est vu comme un grand enfant (sympathique) par les narrateurs, donc jusqu'à un certain point affecté d'un indice négatif, la mère est toujours valorisée, même lorsqu'on fait allusion à des failles possibles chez elle. Fait nouveau dans notre littérature, chez les auteurs masculins du moins, cette mère est valorisée à la fois sexuellement et intellectuellement. 
5. Marie Lalonde est aux antipodes du père : «Son cerveau va si vite parfois qu'elle a peine à se suivre elle-même» (p. 109). Les «têtes» tiennent autant de l'une que de l'autre.

6. Gilles Marcotte, le Roman à l'imparfait, essais sur le roman québécois d'aujourd'hui, Montréal, La Presse, "Échanges», p. 168.

7. «Le roman journal», Liberté, $n^{\circ} 115$, janvier-février 1978, p. 108.

8. «Jacques Godbout et la transformation de la réalité», une entrevue de Donald Smith, Lettres québécoises, $\mathrm{n}^{\circ} 25$, printemps 1982, p. 55.

9. Sur le texte national, il faut relire le célèbre texte de Jacques Godbout, «Ecrire», le Réformiste, textes tranquilles, Montréal, Éditions Quinze, 1975, pp. 147-157, et l'article de Jacques Pelletier, "La problématique nationaliste dans l'oeuvre romanesque de Jacques Godbout», Voix et Images, vol. VI, $n^{\circ} 3$, printemps 1981, pp. 435-451.

10. On doit interpréter la signature «Charles-F. Papineau» a la lumière du passage de la page 19: "Il signe Gregory B. Northridge, en souvenir». Dans les deux cas, c'est l'élément français (françois?) du nom qui a subi une réduction.

11. Je crois avoir identifié ce serpent de mer bicéphale dans un dessin animé contemporain dont le titre est John Blackstar. Cette observation confirmerait une fois de plus l'extrême perméabilité de Godbout à tout ce qui l'entoure.

12. Même si ce dernier fait est moins établi, on en trouve quelques indices assez sûrs dont en particulier les traits américains du père et son enthousiasme de la fin: "Vive la chirurgie!», «A Marie!" (usque ad mare) et «Mes enfants, je propose un toast à l'évolution!» (pp. 149-150).

13. «Texte et idéologie», Poétique, 49, février 1982, pp. 105-125.

14. Observations qui remontent à quelques années déjà et dont je relève un écho plus récent dans la Presse du ler décembre 1982: «Les Canadiens français sont affligés de nombreuses maladies génétiques héréditaires».

15. Louis Hémon, Maria Chapdelaine, Montréal, Fides, 1974, p. 212.

16. Le cliché ne semble pas si connu de Léandre Bergeron dans son Dictionnaire de la langue québécoise, Montréal, VLB éditeur, 1980, p. 485: «Avoir, être une tête à Papineau - Etre très intelligent). Cette méconnaissance de la langue (de ses syntagmes figés en particulier) conjuguée $i l$ l'ignorance de ce qu'est vraiment un dictionnaire a valu a l'auteur des critiques très acerbes dont la plus savoureuse et la plus exhaustive est surement celle de Danielle Trudeau, Léandre et son péché, Montréal, Hurtubise HMH, 1982.

17. Il faut observer que le pluriel commence avec le nombre «deux», la langue française ne connaissant pas la forme duelle.

18. L'impossibilité d'une littérature d'expression française est signifiée différemment par le best-seller du Dr (et non Docteur!) Bonvouloir, «traduit en plusieurs langues, dont la nôtre!» (p. 30), comme si la langue anglaise était garante du succès. Dans $D^{\prime} a m o u r, P . Q$., les déclarations sur la langue étaient moins radicales: "Il ne peut y avoir de littérature bilingue" (p. 153).

19. Le sujet «nous» est d'ailleurs rarement associé à une action ponctuelle au présent.

20. Ces considérations s'inspirent des vues de Mikhail Bakhtine sur le rôle des genres intercalaires dans le plurilinguisme du roman, Esthétique et Théorie du roman, Paris, Gallimard, 1978, p. 141 et suivantes. En plus des deux genres mentionnés plus haut qui constituent l'armature du roman, d'autres genres sont intercalés: extraits de la biographie du Dr Bonvouloir, articles de journal, texte de fiction de François.

21. «Jacques Godbout et la transformation de la réalité», pp. 60 et 61. 